\title{
Limited Urethral Mobilization Technique in Distal Hypospadias Repair : An Overview
}

\author{
Abhi Kumar Chakraborty ${ }^{1 *}$ \\ Sajal Kumar Majumdar ${ }^{1}$ \\ Mirza Kamrul Zahid ${ }^{1}$ \\ Ipsita Biswas ${ }^{2}$ \\ Poritosh Palit ${ }^{1}$
}

'Department of Paediatric Surgery Shaheed Suhrawardy Medical College Dhaka, Bangladesh.

${ }^{2}$ Department of Paediatric Surgery Dhaka Shishu Hospital Dhaka, Bangladesh

*Correspondence to:

\section{Dr. Abhi Kumar Chakraborty}

Assistant Professor

Department of Paediatric Surgery

Shaheed Suhrawardy Medical College

Dhaka, Bangladesh.

Mobile : +88 01712203795

E-mail :abhichakraborty@yahoo.com

\begin{abstract}
Background: Fistula formation is the most common complication with various rates among different techniques of hypospadias repair. The urethral advancement as an one stage technique for repair of hypospadias is considered a good technique. As no new urethral tube is constructed there is no risk of fistula. We assess the outcomes of the Limited Urethral Mobilization (LUM) technique in distal hypospadias repair. Methods: Our study included 20 patients aged upto 12 years who were treated with Limited urethral mobilization technique for distal hypospadias in Shaheed Suhrawardy Medical College Hospital from December 2014 to December 2015. The urethra proximal to the meatus was mobilized for a distance sufficient to allow it to reach the glans tip without tension. Then the urethra was placed in the glanular bed and glanular reconstruction was performed. Results: Three-fold urethral mobilization was sufficient for construction of tension-free urethra-glanular anastomosis. Cosmetically normal looking circumcised penis with ventrally slit meatus achieved in all but one subcoronal case where the most distal of the glans approximation sutures disrupted leading to a minor detachment in the glans. The minor complications includes preputial edema in one patient, two patients had wound infection, one had meatal stenosis which was treated with gentle dilatations. Postoperative urethro cutaneous fistula, retraction of the urethral meatus or recurrent chordee were not observed. Conclusion: Distal hypospadias repair with the LUM technique can be effective with satisfactory cosmetic and functional results. There is no chance for development of urethro cutaneous fistula, a major postoperative complication of other surgical techniques of creating a neourethra. Postoperative management is simple and a brief hospital stay is sufficient.
\end{abstract}

Key words: Distal hypospadias; Limited urethral mobilization; Outcome.

\section{INTRODUCTION}

The majority of hypospadias cases are distal varieties with an incidence of $75 \%{ }^{1}$. The main goals in hypospadias surgery are penile straightening, urethroplasty, meatoplasty, glanduloplasty,scrotoplasty and skin coverage. Various surgical techniques were developed to achieve these objectives ${ }^{2}$.

Despite the versatility and the obvious surgical advances of different techniques of hypospadias repair, urethrocutaneous fistula is the most common complication, the reported incidence being as high as $5 \%-15 \%{ }^{3,4}$. The incidence of which has been used to evaluate the success of hypospadias repair.

Advancing the urethra for hypospadias repair was first introduced in 1898 by Beck ${ }^{5}$. However, this procedure was not consistently successful because the urethra was not mobilized widely and many patients had chordee ${ }^{6}$. 
Belman reported successful results with a technique for hypospadias repair which involved wide urethral mobilization and advancement ${ }^{7}$.

The elasticity and rich proximal blood supply of the urethra have been demonstrated anatomically and many studies show that it may be safely mobilized in hypospadias repair ${ }^{8}$.

The extent of mobilization varies from minimal distal mobilization to more extensive dissection down to the bulbar urethra ${ }^{2,8}$. Urethral mobilization and meatal advancement with many modifications was found to be a safe procedure in distal hypospadias repair ${ }^{9-12}$

We present our experience with limited urethral mobilization for distal hypospadias repair with the hypothesis that maximizing the use of native urethra would minimize complications.

\section{MATERIALS AND METHODS}

The population under study were male patients younger than 12 years suffering from distal hypospadias with no or mild chordee underwent LUM urethroplasty between December 2014 and December 2015 in the Department of Pediatric Surgery, Shaheed Suhrawardy Medical College Hospital. They had no history of previous hypospadias repair or circumcision. Informed signed consent was obtained for all patient's guardian.

Patient's age, meatal location, presence of ventral curvature, operation time, duration of follow up, post operative evaluations and complications were determined.

Surgical technique : All the operations were carried out under caudal anesthesia.

A 5/0 traction suture was placed at the tip of the glans penis. The distance between the urethral meatus and the glans tip was measured and recorded.

A urethral stent 6 to $10 \mathrm{Fr}$ according to age of the patient was introduced into the bladder. A circumcising incision with Ushaped ventral extension proximal to the hypospadiac meatus was made. The penile skin was degloved down to the penoscrotal junction, releasing any cutaneous chordee.The meatus was circumscribed and the urethra proximal to the meatus was mobilized circumferentially for a distance that sufficient to allow the urethra to reach the tip of the glans without tension after trimming the distal urethra in an oblique fashion. The length of the urethral mobilization was measured and recorded.

The ventral glans was incised deeply into the corpora cavernosa followed by excision of excess mucosa on both sides and the glans wings were mobilized laterally. The glandular bed was prepared and then the mobilized urethra was placed in it. The dorsal lip of the urethra was sutured to the glans tip with interrupted $6 / 0$ polyglactin sutures.

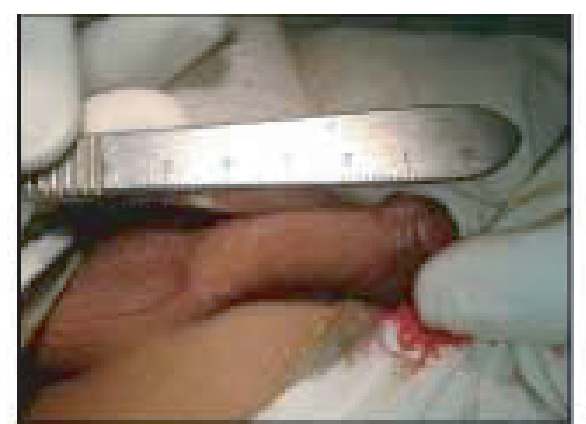

Figure 1: Distance between the urethral meatus and tip of the glans measured and recorded.

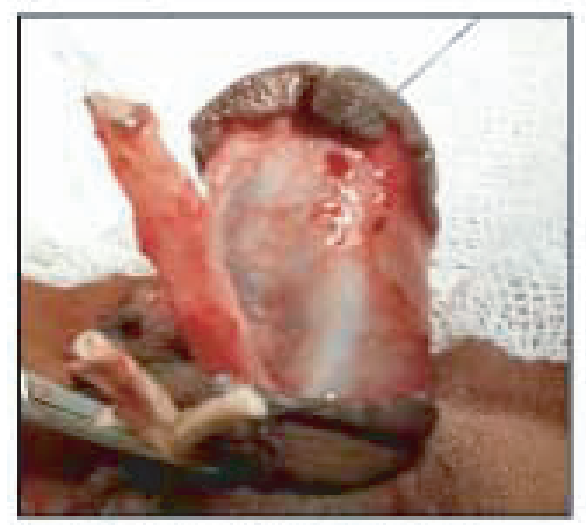

Figure 2 : Mobilization of urethra.

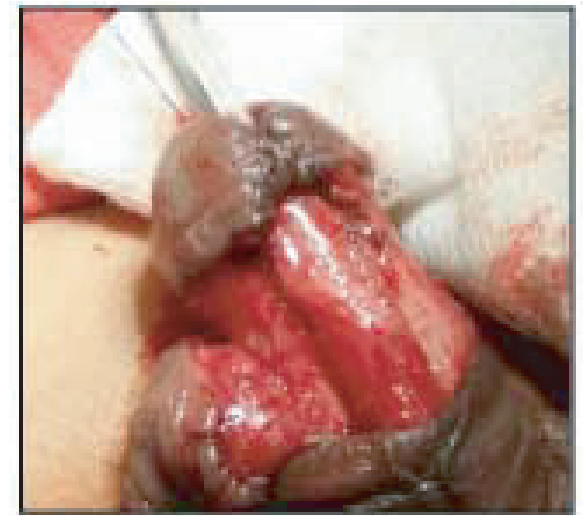

Fig III: Urethra placed in prepared glanular bed.

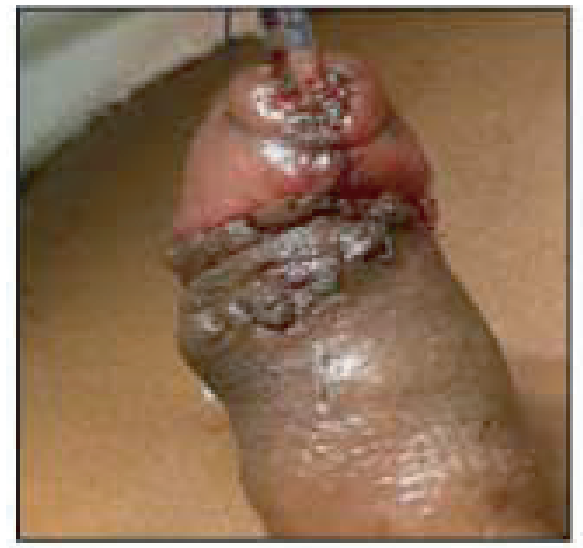

Fig IV : At the end of the operation. 
Glans wings were wrapped around the urethra as in the normal configuration and sutured with 2 layers of interrupted $6 / 0$ polyglactin sutures. The deeper layer secured the urethra to the glanular tissue and the superficial layer approximated the glans wings. The anterior lip of the urethra was secured to the glans with interrupted sutures. A few $6 / 0$ polyglactin interrupted fixation sutures were placed through the tunica albuginea of corpus spongiosum to the corpora cavernosa.

The penile tourniquet, which was used intermittently during dissection was removed when the glans reconstruction was completed. Circumcision was performed in all cases. A compression dressing was applied. The stent was secured with a glanular traction suture was left in place for 5 days.

On the post-operative 5th day the diversion was removed and the patient was discharged after voiding. All patient received parenteral cefuroxime started intraoperatively and continue for three days, followed by oral preparation for next 5 days with analgesics.

On the post-operative 15 th day, urethral calibration was performed with 6-10 Fr stent according to the age of the children.

Patients were called for office visits after 1 month, 3 month of operation and then 3 monthly or before if any complication occured to evaluate the cosmetic appearance and functional results. Cosmesis was evaluated with a slit-like meatus at the tip of the glans with conical glans and lack of chordee, while function was evaluated with a good stream of urine.

\section{RESULTS}

Follow up period ranged from 3 to 18 months (Mean 9 months). Total 27 hypospadias patients were treated in the hospital during the study Period. Out of them 20 (74\%) were distal variety who were enrolled for this study. Age range of the patients were 3 years to 12 years (Mean 7 years 6 months). Two patient with distal penile variety had mild chordee. After degloving the penile skin, the chordee was corrected. Results are summarized in Table 1 and 2.

The mean distance required for carrying the urethral meatus to the glans tip was $7.6 \mathrm{~mm}$ ( $5 \mathrm{~mm}$ to $12 \mathrm{~mm})$ and the length of the mobilized urethra was $15 \mathrm{~mm}$ to $37 \mathrm{~mm}$ (Mean $23.9 \mathrm{~mm}$ ). The ratio of the length of the mobilized urethra to the distance required for carrying the urethral meatus to the glans tip was 3.1(2.7 to 3.2). Mean operation time was 63 minutes (55 minutes to 70 minutes).

None of the patients had major complications. The minor complications which were treated conservatively with satisfactory result. includes preputial edema in one patient $(5 \%)$ two patients had wound infection, one had meatal stenosis who was followed up with reduction in urinary flow calibration in the early post-operative period and the problem was solved with gentle dilatations for two weeks.

All the remaining patients with successful repair had good urinary flow and void with a single stream.
A cosmetically normal looking circumcised penis with ventrally slit meatus at the conical shaped glans of a straight penis was achieved in all but one subcoronal case where the most distal of the glans approximation sutures disrupted leading to a minor detachment in the glans. Although, this condition was a blemish for cosmetic appearance, no functional abnormality occurred. No urethra-cutaneous fistula, retraction of the urethral meatus or recurrent chordee were observed.

Neourethral caliber was satisfactory in the follow up period of this study.

Table I: Preoperative patients characteristics.

\begin{tabular}{llrr} 
Variables & & No & $\%$ \\
Patients age & & & \\
& $3-5 \mathrm{yrs}$ & 04 & $20 \%$ \\
& $>5-10 \mathrm{yrs}$ & 15 & $75 \%$ \\
Meatal site & $>10 \mathrm{yrs}$ & 01 & $5 \%$ \\
& & & \\
& Glanular & 2 & $10 \%$ \\
& Coronal & 07 & $35 \%$ \\
& Sub coronal & 11 & $55 \%$ \\
& & & \\
& Glanular & 00 & 00 \\
& Coronal & 00 & 00 \\
& Sub coronal & 02 & $0 \%$ \\
\hline
\end{tabular}

Table 2 : Postoperative Complications.

\begin{tabular}{llc} 
Complication & No & $\%$ \\
\hline Preputial edema & 01 & $05 \%$ \\
Wound infection & 02 & $10 \%$ \\
Meatal stenosis & 01 & $05 \%$ \\
\hline
\end{tabular}

\section{DISCUSSION}

Urethral mobilization has been performed for more than a century in hypospadias repair. It is known that there is a fine network between the urethral branch of the internal pudendal artery and terminal branches of the dorsal penile artery, which creates an important vasculature for the urethra. Thus, the fear of devascularization due to urethral mobilization seems to be unfounded $^{2}$. On the otherhand, the natural elasticity of the urethra is the main factor for using this technique and the continuing growth of the spongy urethra keeps pace with the general growth of the child ${ }^{8,12}$.

In the present series, the mean mobilized length of the urethra was $23.9 \mathrm{~mm}$ ( $15-37 \mathrm{~mm}$ ) and the distance necessary to carry the urethral meatus to the glans tip was $7.6 \mathrm{~mm}$ ( 5 to $12 \mathrm{~mm}$ ).

We noticed that urethra was advanced until $0.8 \mathrm{~cm}$ in patient with distal hypospadias aged from three to five years and 1.2 $\mathrm{cm}$ in those aged from more than five years to ten years. 
Awad MMS found that urethra can be advanced until $0.5 \mathrm{~cm}$ in patient with distal hypospadias aged from two to five years, about $1 \mathrm{~cm}$ in those aged from more than five to ten years and about $2.5 \mathrm{~cm}$ for those more than ten years ${ }^{13}$. The length of urethral mobilization reported is also typically limited, as in the study by EL Saddi MM in which the urethra was not mobilized more than $1.5 \mathrm{~cm}^{14}$.

We prefer it to be done for repair of distal penile hypospadias as the best choice keeping in mind the relation between the patient age and the mobilized urethral distance needed.

Our data revealed that 2.7 to $3: 2$ (Mean 3.1) fold urethral length mobilization would be adequate to preclude chordee. Interestingly, the data from the present study reveal that a shorter mobilization length can also provide secure urethraglanular anastomosis without chordee and closely resembles the findings of Elemen et al and Hammouda et al who found that a three fold urethral mobilization was sufficient for repair of anterior hypospadias ${ }^{2,10}$.

We found that about three-fold penile urethral length mobilization allows tension-free urethral anastomosis in the case of anterior hypospadias. Atala described four-to-five-fold urethral mobilization to gain tension-free urethral anastomosis, this difference may be due to their inclusion of mid-shaft hypospadias 6 .

Postoperative urethral fistula was not recorded in our series, as there is no anastomosis between the urethra and the neourethra, which can be a potential site for leakage and fistula formation $^{10}$.

El saady MM claimed that the cause of stenosis was due to inadequate urethral opening of the glans ${ }^{14}$. We think that trimming the distal end of the urethra in oblique manner makes it wider and thereby excising the dystrophic part which has no urethelium and may cause post operative stenosis or even wound sloughing. Wide dissection of glans makes space to lay the neourethra, also limits chances for stenosis formation. We observed only one case on $3^{\text {rd }}$ postoperative week and the stenosis was relieved by dilatation twice weekly in two weeks by passage of JMS feeding tube without anaesthesia.
Cosmetically normal looking circumcised penis with ventrally slit meatus at the conical shaped glans were achived in all but one case the most distal of the glans approximation sutures disrupted leading to a minor detachment in the glans. This patient was in early cases in our series. We believe that this condition was due to inadequate preparation of the glandular bed to accommodate the urethra. Although this condition was a handicap for cosmetic appearance, no functional abnormality occurred.

Our study has several limitations. We had small sample size with only short-term followup. Recent reports, including that of Seibold et al show excellent long-term outcomes of urethral mobilization techniques ${ }^{15}$. That study included 99 patients with a followup of 5 years or greater. Ninety seven percent of patients had an excellent cosmetic and functional result based on the validated HOSE (Hypospadias Objective Scoring Evauation) system.

The advantages of this technique are- making a new urethra in one stage, fistula formation is not expected, glans shape is not a factor (Flat, conical, deeply grooved), ability to remove dysplastic ventral elements, if present; limited mobilization of urethra with preservation of blood supply, short duration of hospital stay, can be performed in previous circumcision and hypospadias surgery patient ${ }^{1,8}$.

Proper urethral mobilization, deep interballanitic incision and wide dissection of the glans laterally are essential to avoid complications such as curvature, glanular disruption and meatal retraction or stenosis.

Fine interrupted sutures between the tunica albuginea of corpus spongiosum to that of both corpora cavernosa will contribute to urethral stability.

\section{CONCLUSION}

Although perhaps only useful in distal hypospadias repairs, the LUM technique seems to be a good method with satisfactory cosmetic and functional results. About 3 fold urethral mobilization length is adequate to prevent chordee and achieve tension-free urethra-glanular anastomosis.

\section{DISCLOSURE}

All the authors declared no competing interest. 


\section{REFERENCES}

1. Alkan M, Oguzkurt P, Ezer SS, Ince E, Hicsonmez A: Evaluation of the results of eccentric circummeatal-based flap with combined limited urethral mobilization technique for distal hypospadias repair. J Pediatr Urol. 2008;4:206-209.

2. Elemen L, Tugay M : Limited Urethral Mobilization Technique in Distal Hypospadias Repair with Satisfactory Results. Balkan Med J. 2012; 29: 21-25.

3. Sozubir S and Snodgrass W : A New Algorithm for Primary Hypospadias Repair Based On Tip Urethroplasty. Journal of Pediatric Surgery 2003;38(8):1157-1161

4. Awad MMS , Tolba AM, Saad KM, Zaghlol MR,Rozigque E, Gharib OH, Khalil SA: What is the best choice for repair of distal penile hypospadias: The tubularized incised plate urethroplasty or anterior urethral advancement technique? Indian J Plast Surg. 2007;40(2):182-188

5. Beck C: A new operation for balanic hypospadias. NY Med J. 1898; 67: 147.

6. Atala A: Urethral mobilization and advancement for midshaft to distal hypospadias. J Urol. 2002;168: 1738-1741.

7. Belman AB. Urethroplasty. Society for Pediatric Urology Newsletter 28 December 1977.

8. Koenig JF., Kottwitz M and McKenna PH :Urethral Mobilization for Distal and Mid Shaft Hypospadias with Chordee. The journal of Urology. 2013; 190:1545-1549.

9. El Saadi M.M. , Tolba A M, Sarhan A.E: Anterior Urethral Advancement in Repair of Hypospadias: A Modification of the Technique. Annals of Pediatric Surgery. 2010; 6(1):18-21.

10. Hammouda HM, Hassan YS, Abdelateef AM, Elgammal MA : New concept in urethral advancement for anterior hypospadias. J Pediatr Urol. 2008; 4: 286-289.

11. Roodsari SS, Mulaeian M, Hiradfar M. Urethral advancement and glanuloplasty with V flap of the glans in the repair of anterior hypospadias. Asian J Surg. 2006;29:180-184

12. Haberlik A, Schmidt B, Uray E, Mayr J : Hypospadias repair using a modification of Beck's operation: Followup. J Urol. 1997; 157: $2308-2311$.

13. Awad MS. Urethral advancement technique for repair of distal penile hypospadias: A revisit. Indian J Plast Surg. 2006; 39: 34-38.

14. El Saadi MM. Anterior urethral advancement technique for repair of hypospadias: Experience with 55 patients. Zagazig Med Assoc J. 1995; $1: 31-37$.

15. Seibold J, Werther M, Alloussi S,Gakis G,Schilling D, Colleselli D et al: Objective long-term evaluation after distal hypospadias repair using the meatal mobilization technique. Scand J Urol Nephrol. 2010; 44: 298 -303. 